\title{
Addressing complexities in self-regulated learning: a focus on contextual factors, contingencies, and dynamic relations
}

\author{
Adar Ben-Eliyahu • Matthew L. Bernacki
}

Received: 24 January 2015 / Accepted: 30 January 2015 /

Published online: 26 February 2015

(C) Springer Science+Business Media New York 2015

\section{Introduction}

The conceptualization of self-regulated learning (SRL) has been profoundly articulated in recent special issues focused on the similarities and distinctions between SRL and related phenomena like self-regulation, metacognition, and motivation as well as the development of new environments and methods that improve SRL research (Alexander 2008; Azevedo 2005a, 2005b, 2007; Azevedo and Hadwin 2005; Greene and Azevedo 2010; Gress and Hadwin 2010; Järvelä and Hadwin 2013; Molenaar and Järvelä 2014; Perry 2002; Winne and Baker 2013). These special issues have examined how the affordances of (usually computer-based) learning environments can provide the means to observe phenomena described in SRL models and to empirically test theoretical assumptions (Azevedo 2005b; Azevedo and Hadwin 2005; Efklides 2011; Winne and Hadwin 2008; Winne 2010, 2011; Zimmerman and Schunk 2008, 2011). In this special issue, we tighten the focus of this ongoing discussion by focusing on three key features of SRL frameworks: contextual factors, contingencies, and dynamic relations. Contextual factors encompass features of the learning environment, the learner, and their interaction (Efklides 2011). Contingencies refer to instances where one event occurs in the context of another (Winne and Hadwin 2008; Winne 2010, 2011) - a critical assumption of process models of SRL and the basis for research on sequential and temporal characteristics of SRL (Molenaar and Järvelä 2014). Dynamic relations occur when two or more SRL processes influence one another (Winne and Hadwin 2008; Zimmerman and Schunk 2011).

The contributors of this special issue are leading international researchers who examine how contextual factors influence SRL (Ben-Eliyahu and Linnenbrink-Garcia 2015; McCardle and Hadwin 2015), how contingent metacognitive processes influence achievement (Binbarasan-Tüysüzoğlu and Greene 2015), and how SRL processes dynamically influence one another as learning unfolds (Bernacki et al. 2015; Lichtinger and Kaplan 2015). Two commentaries identify the cross-cutting themes of the special issue (Karabenick and Zusho 2015) and appraise the alignment between the theoretical assumptions, research designs, and

\footnotetext{
A. Ben-Eliyahu $(\bowtie)$

Faculty of Education, University of Haifa, 199 Aba Hushi Ave., Haifa 3498838, Israel

e-mail: adarbe@edu.haifa.ac.il

M. L. Bernacki

Department of Educational Psychology \& Higher Education, University of Nevada, Las Vegas, Las Vegas, NV, USA
} 
analyses in these and other studies of SRL (Dent and Hoyle 2015). In this introduction, we define contextual factors, contingencies, and dynamic relations, chronicle their emergence as key components of SRL theories, and consider how the articles from this special issue reflect these themes. We propose the integrated Self-Regulated Learning (iSRL) model, which draws on work from the social and clinical sciences (Gross 2008; Gross and John 2003; Hoyle 2010; Schmeichel and Baumeister 2004) to situate our discussion of SRL within the broader lifecontext of the individual (Bowers et al. 2011; Bronfenbrenner 1977, 1994; Bronfenbrenner and Ceci 1994) and use the iSRL model to demonstrate how SRL processes in a learning task can be affected by factors that are outside of the immediate context (e.g., neighborhood, family, school, course) where SRL is observed. We also consider the time scale at which SRL phenomena are observed and propose that the use of a common time scale can help reconcile research on a single SRL phenomenon observed using differently focused methods of observation. This organization, adapted from cognitive science (Newell 1990) can further assist researchers in obtaining the alignment that Dent and Hoyle (2015) underscore as critical in SRL research.

\section{Context}

From the earliest conceptualizations, "context" has appeared as a key component of SRL frameworks (e.g. Pintrich 2000; as "task in context" in Boekaerts and Niemivirta 2000; as "learning task" in Winne and Hadwin 1998; as "environment" in Zimmerman 2000). New frameworks have placed increased focus on how context, its evolution, and its interaction with individual characteristics influences SRL (Efklides 2011; Winne 2010). The "task" generally refers to the immediate learning task defined at the initiation of the SRL process (Winne and Hadwin 1998), whereas the learning environment refers to broader characteristics such as the learning space (e.g., school-based course or museum) or features (e.g., liked versus disliked discipline). To empirically examine these direct and indirect effects requires that the task be operationally defined and that features of the task environment are accounted for and incorporated into research designs. In this special issue, we extend the consideration of contextual factors beyond the immediate characteristics of the learning task to include characteristics of the larger learning context in which the task is embedded. For example, studies by BenEliyahu and Linnenbrink-Garcia (2015) and McCardle and Hadwin (2015) examine contextual factors at the course-level, as well as the more distal factors that may also affect how individuals self-regulate their learning.

\section{Contingency}

As with contextual factors, contingent relationships were critical components of early process models of SRL that assume events in one cycle of SRL are contingent upon prior steps or prior cycles (e.g., Winne 1985; Winne and Hadwin 1998; Zimmerman 1989, 2000). Contingencies refer to IF - THEN scenarios where a learner consciously determines that, in response to the occurrence of a specific SRL event (i.e., the "IF"), a second event or process should be initiated (i.e., the THEN; Winne 2010). For example, reading a dense passage may be identified as challenging by making a metacognitive monitoring judgment. In light of this judgment, the learner selects a metacognitive control strategy, like note taking. Here, an observation (i.e., the reading is dense) is made during the monitoring process, which cues the contingent selection of the note taking strategy selection (i.e., "IF I encounter a dense reading passage, THEN I should be sure to take notes so that I can elaborate on the most important segments of the reading"). 
Refinements in the early process models place renewed emphasis on contingent relationships that can be observed when SRL events are examined as they unfold over time. Research methods have emerged to examine contingent relations (e.g., Winne 2011) and empirical work has begun to emerge examining the occurrence and outcomes of contingent events (cf. Molenaar and Järvelä 2014). In this issue, Binbarasan-Tüysüzoğlu and Greene (2015) use judgments of learning made when thinking aloud to test assumption of the COPES model of SRL (Winne 1997, 2010, 2011, 2014; Winne and Hadwin 1998, 2008) and examine contingencies between metacognitive monitoring and control process and their effects on academic performance.

\section{Dynamic relations}

Models that conceptualize SRL as an iterative, cyclical process are, by rule, dynamic models in that any activity that occurs within one cycle can affect activities that follow within that cycle, and any activity within subsequent cycles (Boekaerts and Niemivirta 2000; Pintrich 2000; Winne and Hadwin 1998; Zimmerman 2000). For instance, Zimmerman's (1989; 2000) social cognitive model of SRL formalized dynamic relations amongst components of SRL in diagrammatic fashion as feedback loops between two processes and Butler and Winne (1995) acknowledged "recursive" relations where monitoring activities prompt cognitive engagement and feedback that are then regulated as further cognitive engagement is monitored. More recently, Winne and Hadwin (2008) built upon their initial COPES model (Winne and Hadwin 1998) by further refining the relations they proposed exist between motivational constructs and self-regulatory processes, and Zimmerman and Schunk (2008; 2011) further clarified the hypothesized role of specific motivational constructs within SRL as precursors, mediators, and outcomes of SRL processes. In this issue, two articles examine dynamic relations in very different specificities. Bernacki et al. (2015) use embedded questionnaires and trace methodologies to examine minute-to-minute relationships between selfefficacy judgments, help seeking, and problem-solving attempts, whereas Lichtinger and Kaplan (2015) employ classroom observations, strategy traces, and interviews to examine dynamic relations between learners goals, affect, and actions during classroom learning activities.

\section{Methodology and design choices}

As theories of SRL have undergone refinement, so too have the methods researchers have employed to investigate SRL phenomena. Boekaerts (1997, 1999; Boekaerts and Corno 2005; Boekaerts and Cascallar 2006), Winne (2005, 2010, 2014; Winne and Baker 2013; Winne et al. 2011) and others (Azevedo et al. 2010; Bannert et al. 2013; Kinnebrew et al. 2013a, b; Wirth and Leutner 2008) have periodically assessed the degree to which refinements in theory have led researchers to examine SRL phenomena with greater specificity, and how new research methods might afford new opportunities for further theoretical refinement. Like the authors of these reviews, we contend that statistical methodologies required to test theorized relationships among SRL processes have been available to sufficiently skilled researchers for many years. However, researchers' ability to collect data that are sufficiently precise and comprehensive to facilitate these statistical analyses remains a challenge. For example, dynamic relations can be examined with cross lagged path analyses, but only so long as quantitative, longitudinal data on two related constructs over three or more periods are available. Contingent relations can be examined using conditional 
probabilities (Winne 2010), but require that fine-grained data be collected in sequence so that the likelihood that one event follows another can be gauged. Research questions examining the effects that contextual factors have on SRL can be assessed via comparison of structural models, but require that two identical data collections be conducted with the same population in different contexts.

Methodologies common to SRL research include self-report questionnaires (Ainley and Patrick 2006; Karabenick et al. 2012; Pintrich 2004), think-aloud protocols (Azevedo 2005b; Greene et al. 2011), microanalysis (Cleary 2011) and qualitative classroom observation methods (Boekaerts and Corno 2005; Corno 1995; Perry and Rahim 2011). Trace methodologies from think aloud protocols (Greene, Robertson, and Costa 2011) and sequenced, computer-generated logs of learner behaviors are increasingly common (Aleven et al. 2010; Azevedo et al. 2010; Graesser and McNamara. 2010; Greene and Azevedo 2010; Kinnebrew et al. 2013a; Molenaar and Järvelä 2014; Sha, Looi, Chen, Seow, and Wong 2012; Winne and Hadwin 2013). The majority of early studies of SRL employed a single approach to observing SRL and thus have been classified as assessing SRL aptitudes or self-regulatory events that unfold during learning (Winne and Perry 2000). However, recent approaches have emphasized the collection and triangulation of multiple sources of cognitive, affective, metacognitive, and motivational data (see Azevedo et al. 2013).

Many have called for the use of multiple methods to more fully account for features of learning tasks and SRL processes (e.g., Boekaerts and Corno 2005; Schraw 2010; Winne 2010). In this special issue, we intentionally include papers that demonstrate how complementary methods can be employed to provide insight on dynamic or contingent relations amongst phenomena, and how context influences SRL processes. For example, individualized case studies, interviews, and observations (Lichtinger and Kaplan 2015) and fine-grained assessments of motivation and behavior (Bernacki et al. 2015) can be used to examine dynamic relations between motivational and metacognitive processes as they occur over iterations of the SRL cycle. Binbarasan-Tüysüzoğlu and Greene (2015) applied a valenced coding scheme to think aloud protocol transcripts and contingency analysis to examine the contingent relationship between metacognitive monitoring judgments and subsequent metacognitive control while learning with hypermedia. Ben-Eliyahu and Linnenbrink-Garcia (2015) administered parallel self-report surveys anchored to specific learning contexts (i.e. courses) within subjects to assess the influence of learning context on SRL processes. McCardle and Hadwin (2015) also examined learning in a specific context and employed a mix of qualitative open-ended questions and self-report surveys that identify the task as an explicit referent in order to develop a rich portrayal of the SRL process within a specific context. Taken together, these articles demonstrate both the distinct advantages of individual methods for assessing relations among specific SRL phenomena, and that it is often necessary to combine multiple methods in order to accommodate the complex structure of the SRL process. In their commentaries, Karabenick and Zusho (2015) and Dent and Hoyle (2015) consider how the approaches taken by these researchers address questions about context, contingency, and dynamic relations. They critique the theoretical assumptions that prompted each empirical approach, and suggest how researchers might conduct future studies that addresses complexities inherent in theories of SRL. Dent and Hoyle further suggest an organizational framework to facilitate the alignment of research methods to theoretical assumptions. If researchers adopt their recommendations on reporting, this framework can also improve the synthesis of findings across studies of SRL. In addition to demonstrating how a wider range of methodological approaches improves assessment of SRL, we propose that taking a wider lens to the conceptualization of the context 
where SRL occurs can help us better understand how individuals self-regulate their learning.

\section{Incorporating social and clinical psychological lenses to broaden "classic" SRL theory}

Whereas SRL is conceived of as a loosely sequenced set of specific processes that occur in an explicit task context, the SRL process is conducted by an individual who also regulates internal and external distractions, and at additional levels outside the immediate context of the learning task. To understand how regulation in response to phenomena outside the learning task (e.g., unstable home environment) influences SRL, we pose an integrated model of SRL (iSRL) that demonstrates how all SRL processes occur within a hierarchically nested context. This hierarchical structure is best understood through an ecological assets perspective (Bowers et al. 2011; Bronfenbrenner 1977; Bronfenbrenner and Ceci 1994). In the iSRL model, we demonstrate how self-regulatory capacity is limited via depletion (e.g., ego depletion; Schmeichel and Baumeister 2004), extending the notion that SRL is a finite capacity (Winne 2010) beyond the immediate learning task and context, as described below.

\section{Self-regulatory capacity is a limited resource}

Self-regulation, whether directed towards a specific learning task, as in SRL, disciplines (e.g., science or humanities), or more broadly in school or home, requires personal resources. This is especially true when self-regulation is an active undertaking characterized by the use of executive functioning (Schmeichel and Baumeister 2004; Winne 2011). When regulatory resources are used, they become depleted in the same way that muscles fatigue when used. Schmeichel and Baumeister (2004) therefore refer to self-regulatory strength as a deplete-able resource, or as ego depletion. This form of depletion was recognized by Winne (2011) with regard to the limited capacity of cognition; implicit SRL requires less cognitive load than explicit SRL. Thus, even working memory, when applied intentionally, is a limited resource (Winne 2011, p. 16):

A learner in biology class is (hopefully) not attending to dust motes floating in the air, wondering why an attractive girl didn't accept his invitation to a party, or mentally imaging a game-winning slap shot. These and other ideas are available but not focal in working memory.

In this vignette, a learner's attempts to self-regulate learning compete with a broader set of cognitive processes that also vie for attention. Thinking about a party uses the same cognitive resources as learning biology, which depletes the working memory resources that can be applied to the biology task. Only when the learner is solely focused on biology is it accurate to consider SRL processes alone. But in reality, it is more appropriate to conceptualize SRL as co-occurring with other behavioral, cognitive, and emotional processes, as depicted here. There is a scarcity of empirical work that examines how individuals regulate their learning and also accounts for additional cognitive processes unrelated to learning and their interactions. In this special issue, Ben-Eliyahu and Linnenbrink-Garcia examine how the regulation of emotions in one's favorite and least favorite classes are related to SRL processes in these distinct contexts.

Self-regulated learning occurs in a hierarchical context

SRL has been theorized and studied primarily within a discrete learning task (e.g., science learning using hypermedia; Azevedo 2005b) or in course-specific-settings (e.g., Pintrich et al. 
1993; Wolters and Pintrich 1998). The level of specificity implicit in these methodological approaches illustrates researchers' distinct conceptualizations of SRL as it occurs in tasks, courses, or academics. Learning events are observed within a task, which is assigned within a course in a discipline at an academic institution (see Fig. 1). When a researcher's focus is narrow (e.g., examining events in a brief task), it is possible to observe how SRL unfolds. These investigators use methods that produce precise, sequential accounts of events within the task that are critical to observing contingencies and dynamic relations among learning events (Winne and Perry 2000). Most often, however, narrowly focused designs tend to ignore potentially important factors that are visible when focus is broadened to account for learner's individual differences and features present in broader layers of task context. Such broader layers include not only the discipline (e.g. math) and school (e.g., low achieving, urban high school) but also the learners' social-cultural (e.g., family composition) and even broader contexts (e.g., governmental policies). The occurrence of and relations between SRL events in a learning task can be influenced by these superordinate contextual factors; a teenager who had to work late the night before may have less energy to devote when learning the next morning. Thus, the most ecologically valid models of SRL must account for the unique and interactive influence of these seemingly distal factors. In the iSRL model (Fig. 2), we apply an ecological assets perspective in an attempt to illustrate how learners can be influenced not just by the features of the hierarchically nested learning tasks we observe, but also by the ecology of factors that hold influence in their lives (i.e., self, school, neighborhood, educational policies).

Self-regulated learning occurs within an ecological system

As depicted in Fig. 2, SRL does not occur independently of regulatory processes unrelated to learning (Hoyle 2010; Kaplan 2004; Schmeichel and Baumeister 2004). For example, social relationships and non-academic situations influence one's capacity to engage academically; any learning task in which a student self-regulates occurs within a classroom or school context that exists in a neighborhood, within a country's policies, and at a particular time. In applying an ecological assets perspective, at the broadest level, a country's contemporary culture and policies influence opportunities to learn. This shapes learning through provision of resources and technology that influence the task conditions of more specific learning tasks (e.g. composition of essays by hand, with a typewriter, using word processing software). Intermediate influences include individuals and environments within the concentric circles like one's city, neighborhood, and family (Bowers et al. 2011; Bronfenbrenner 1977; Bronfenbrenner and Ceci 1994). These factors have independent influence on the individual, and can also mediate the effects of other factors. Bronfenbrenner (1977) described mesosystem

\begin{tabular}{|c|c|c|c|c|c|c|c|c|c|c|c|c|c|c|c|c|c|}
\hline \multicolumn{18}{|c|}{ School-based/Academic } \\
\hline \multicolumn{10}{|c|}{ Science } & \multicolumn{8}{|c|}{ Math } \\
\hline \multicolumn{5}{|c|}{ Biology } & \multicolumn{5}{|c|}{ Chemistry } & \multicolumn{4}{|c|}{ Algebra } & \multicolumn{4}{|c|}{ Geometry } \\
\hline 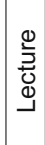 & 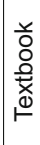 & 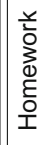 & 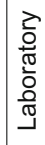 & 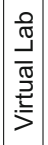 & 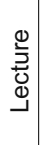 & ठо & 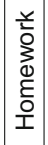 & 。ِ & 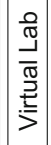 & 0 & 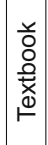 & 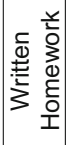 &  & 憘 & 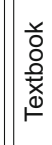 & 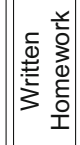 &  \\
\hline
\end{tabular}

Fig. 1 A hierarchical depiction of the grain-sized levels of learning 




Fig. 2 An illustrative depiction of the integrated Self-Regulated Learning (iSRL) model that incorporates a hierarchical perspective of self-regulation through an ecological assets lens incorporating different contextual levels and grain-sizes of learning

as the mechanism through which different features influence each other. Via mesosystems, demands from co-occurring microsystems like learning tasks in that day's history class or relations with peers present in class can deplete (or enhance) one's capacity to regulate during problem solving in math class.

At a societal level, the effect of environmental factors on students' capacity for learning is well understood. For example, federal-level initiatives in the U.S. and other countries (e.g., NoKidHungry; Augustine-Thottungla et al. 2013) acknowledge that students who lack adequate nutrition cannot perform in schools, and address this exosystem-level challenge (i.e., Fig. 2 and Bronfenbrenner 1977) by providing breakfasts for students. Understood vis-à-vis the regulatory strength perspective, a hungry child is regulating their hunger and their ability to learn is thus depleted. To offset this depletion, an intervention (i.e., school breakfast program) must be delivered to replenish their regulatory capacity. An important line of work investigates school climate or neighborhood characteristics and their relations to school achievement (McCoy, Roy, and Sirkman 2013). These studies cross spheres of influence by investigating how exosystems and microsystems are interrelated. In this special issue Ben-Eliyahu and Linnenbrink-Garcia (2015) investigate mesosystems by considering the person by context interaction, while Lichtinger and Kaplan also qualitatively identify contextual factors that influence how SRL unfolds for different individuals.

In the iSRL model, we posit that there are mutual and dynamic influences between one's ecological assets and one's ability to learn because the interaction between one's environment and learning depends on the extent to which non-learning aspects deplete regulatory strength. For example, a student who wakes up to an unstable home environment constantly uses her regulatory strength to cope with day-to-day hardships (e.g., poor relations with a neglectful parent; procuring her own lunch and clothing) not encountered by a typical population. Having had their capacity depleted by other self-regulatory tasks, these students arrive to school with less resources available to self-regulate their learning and are likely to perform poorly during 
learning as a result. Researchers who choose to adopt the iSRL framework to integrate across clinical, educational, and social psychology can better situate how an individual's learning process is influenced by the social and cultural contexts present during learning.

\section{A time scale for situating SRL processes and selecting methods of observation}

SRL is a multifaceted phenomenon that includes components that operate iteratively (process models, Winne and Hadwin 1998; Zimmerman 2000), at multiple levels (micro- and macroprocesses, Greene and Azevedo 2007, 2009) and in different and evolving contexts (Winne 2010). This inherent complexity requires that researchers adopt multiple measurement approaches to observe SRL components that operate differently such that each approach is appropriately aligned to the component under investigation. A meta-analysis of SRL research demonstrates that the choice to observe SRL with online measures (i.e., observations of SRL events, Winne and Perry 2000) versus offline measures (i.e., domain- or course-level surveys of learner tendencies) moderates the strength of relationship observed between SRL processes and achievement measures (Dent and Koenka in press). This difference lends credence to the idea that the level of specificity, or time scale, at which SRL is observed matters when considering how results are interpreted. We propose that, when developing a study to test a specific theoretical assumption, researchers must consider the time scale at which an SRL process is theorized to occur, and choose measurement practices that can observe such phenomena as they occur.

In his work towards establishing Unified Theories of Cognition (1990), Allen Newell attempted to map the architecture of human cognition, and to classify a taxonomy of processes that comprise human thought. As part of this model, Newell developed a system that placed human thought on specific "bands" according to the time scale on which they occur. Using a base-10 system and a logarithmic scale anchored at $10^{\circ}$ equivalent to $1 \mathrm{~s}$, Newell describes events on the biological, cognitive, rational, and social bands. This time scaling approach has been revived in the learning sciences (Nathan and Alibali 2010) and could serve as a useful heuristic for conceptualizing research designs for SRL and explaining why the same process observed at different time scales might yield different results (e.g. learning strategies; Table 1). For instance, SRL events that occur over the range of seconds (i.e. $10^{0}$ ) fall on a cognitive band and include cognitive and metacognitive events, which are typically observed in tasks using trace methodologies (e.g., problem-solving and help-seeking behaviors; Bernacki et al. 2015) and think-aloud protocols (e.g., judgments of learning; Binbarasan-Tüysüzoğlu and Greene 2015). Events on the rational band occur over several minutes to several hours (i.e. $10^{2}$ to $10^{4}$ ), and include patterns of events, changes in learning strategies (e.g., adaptive metacognitive control strategies; Binbarasan-Tüysüzoğlu and Greene 2015), and fluctuations in motivation (change in self-efficacy; Bernacki et al. 2015). These might also include general tendencies or aggregate counts of SRL behavior, as captured in journals (McCardle and Hadwin 2015) or interviews (Lichtinger and Kaplan 2015). Contextual factors in SRL stemming from features of the discipline, course, or school are best observed on a social band; they are relatively enduring features of the environment that, if they evolve, do so slowly over periods of weeks, and months (i.e. $10^{5}$, to $10^{7}$ ). For example, SRL in specific courses assessed via self-report questionnaires (e.g. favorite/least favorite class; Ben-Eliyahu and Linnenbrink-Garcia 2015) or emerge as aggregate summaries of typical SRL behaviors that accrue over an entire course (McCardle and Hadwin 2015). When a specific course is used as a referent, survey approaches may also account for the hierarchical nestedness of a course (Ben-Eliyahu and Linnenbrink-Garcia 2015) or 
Table 1 Measurement Approaches by Time Scale and SRL Component per Study

\begin{tabular}{|c|c|c|c|c|}
\hline \multirow[t]{2}{*}{ Study } & \multirow[t]{2}{*}{ SRL Component (measurement method) } & \multicolumn{3}{|c|}{ Time scale of observation } \\
\hline & & $\begin{array}{l}\text { Cognitive } \\
10^{\circ}\end{array}$ & $\begin{array}{l}\text { Rational } \\
10^{2} \text { to } 10^{4}\end{array}$ & $\begin{array}{l}\text { Social } \\
10^{5} \text { to } 10^{7}\end{array}$ \\
\hline \multirow[t]{3}{*}{$\begin{array}{l}\text { Ben Eliyahu \& } \\
\text { Linnenbrink- Garcia }\end{array}$} & $\begin{array}{l}\text { - Course characteristic (self-reported } \\
\text { favorite/least favorite class) }\end{array}$ & & & $x$ \\
\hline & $\begin{array}{l}\text { - Affective, Cognitive \& Behavioral } \\
\text { Regulation (self-report questionnaires; } \\
\text { referent = general capacity) }\end{array}$ & & & $x$ \\
\hline & $\begin{array}{l}\text { - Learning strategies (self-report questionnaire; } \\
\text { referent }=\text { course) }\end{array}$ & & & $\times$ \\
\hline \multirow[t]{2}{*}{ McCardle \& Hadwin } & $\begin{array}{l}\text { - Perceptions of SRL actions \& strategies } \\
\text { (self-report questionnaire; referent = } \\
\text { recent study session) }\end{array}$ & & $\times$ & \\
\hline & - Weekly Reflections (open ended self-report) & & & $\times$ \\
\hline \multirow[t]{3}{*}{$\begin{array}{l}\text { Bernacki, Nokes- } \\
\text { Malach \& Aleven }\end{array}$} & $\begin{array}{l}\text { - Problem solving behaviors (traces from log } \\
\text { of learning events generated by software) }\end{array}$ & $\times$ & & \\
\hline & - Help seeking behaviors (traces) & $\times$ & & \\
\hline & - Self-efficacy (recurring single self-report item) & $\times$ & & \\
\hline \multirow[t]{4}{*}{ Lichtinger \& Kaplan } & - Learning Strategy use (traced via classwork) & & $x$ & \\
\hline & $\begin{array}{l}\text { - Qualitative classroom observation of } \\
\text { engagement process (observation) }\end{array}$ & $x$ & $\times$ & \\
\hline & $\begin{array}{l}\text { - Stimulated recall interview re: engagement } \\
\text { in classroom learning (interview referring to } \\
\text { a learning task) }\end{array}$ & & $\times$ & \\
\hline & $\begin{array}{l}\text { - General interview re: engagement, learning } \\
\text { in school, self-regulation aptitude (interview) }\end{array}$ & & & $\times$ \\
\hline \multirow{2}{*}{$\begin{array}{l}\text { Binbarasan- Tüysüzoğlu } \\
\text { \& Greene }\end{array}$} & - Judgments of Learning (think aloud protocol) & $\times$ & & \\
\hline & - Learning strategies (think aloud protocol) & & $\times$ & \\
\hline
\end{tabular}

may improve the accuracy and specificity of students' reporting of SRL tendencies (McCardle and Hadwin 2015).

Combining measures that span more than one band provides a rich account of SRL. In their qualitative study, Lichtinger and Kaplan (2015) employ a trans-scale approach (Nathan and Alibali 2010). They examine the SRL of eight elementary school students by observing behavioral events during learning (i.e. cognitive band), tracing the strategies employed on assignments (i.e. rational band), and interviewing students about their past experiences as they relate to purposes for task engagement and self-perceptions of aptitude to self-regulate (i.e., social band). The effort involved in triangulating these methods precludes the use of large, representative samples, but also provides a cross-cutting set of measures that span all bands and allow for inferences as to how SRL processes may dynamically relate to one another over the course of a semester.

\section{Conclusion}

In this issue, we introduce frameworks for considering the hierarchical contexts in which SRL tasks are nested, the influence that more distant layers of context may have on SRL in an 
immediate task, and the varying time scales on which such phenomena should be measured. The empirical studies provide examples of research that considers these complexities in SRL frameworks including contextual factors, contingencies, and dynamic relations. They also illustrate how carefully chosen methods can successfully test corresponding theoretical assumptions. The two commentaries in the issue underscore that incorporating multiple methodologies aligned to the specific SRL components in focus is critical, and that an a priori appraisal of the alignment of between conceptualization and methodology and analysis can produce future research that contributes to our understanding of the complex processes that comprise self-regulated learning.

\section{References}

Ainley, M., \& Patrick, L. (2006). Measuring self-regulated learning processes through tracking patterns of student interaction with achievement activities. Educational Psychology Review, 18(3), 267-286.

Aleven, V., Roll, I., McLaren, B. M., \& Koedinger, K. R. (2010). Automated, unobtrusive, action-by-action assessment of self-regulation during learning with an intelligent tutoring system. Educational Psychologist, 45(4), 224-233. doi:10.1080/00461520.2010.51774.

Alexander, P. A. (2008). Why this and why now? Introduction to the special issue on metacognition, selfregulation, and self-regulated learning. Educational Psychology Review, 20(4), 369-372. doi:10.1007/ s10648-008-9089-.

Augustine-Thottungla, R., Kern, J., Key, J., \& Sherman, B. (2013). Ending childhood hunger: A social impact analysis. Washington: Deloitte.

Azevedo, R. (2005a). Computer environments as metacognitive tools for enhancing learning. Educational Psychologist, 40(4), 193-197.

Azevedo, R. (2005b). Using hypermedia as a metacognitive tool for enhancing student learning? The role of selfregulated learning. Educational Psychologist, 40(4), 199-209.

Azevedo, R. (2007). Understanding the complex nature of self-regulated learning processes in learning with computer-based learning environments: an introduction. Metacognition and Learning, 2(2/3), 57-65.

Azevedo, R., \& Hadwin, A. F. (2005). Introduction to special issue: Scaffolding self-regulated learning and metacognition: implications for the design of computer-based scaffolds. Instructional Science, 33, 367-379.

Azevedo, R., Harley, J., Trevors, G., Duffy, M., Feyzi-Behnagh, R., Bouchet, F., \& Landis, R. S. (2013). Using trace data to examine the complex roles of cognitive, metacognitive, and emotional self-regulatory processes during learning with multi-agent systems. In R. Azevedo \& V. Aleven (Eds.), International handbook of metacognition and learning technologies (pp. 427-449). New York: Springer.

Azevedo, R., Moos, D. C., Johnson, A. M., \& Chauncey, A. D. (2010). Measuring cognitive and metacognitive regulatory processes during hypermedia learning: issues and challenges. Educational Psychologist, 45(4), $210-223$.

Bannert, M., Reimann, P., \& Sonnenberg, C. (2013). Process mining techniques for analysing patterns and strategies in students' self-regulated learning. Metacognition and Learning, 9, 161-185. doi:10.1007/ s11409-013-9107-6.

Ben-Eliyahu, A., \& Linnenbrink-Garcia, L. (2015). Integrating the regulation of affect, behavior, and cognition into self-regulated learning paradigms among secondary and post-secondary students. Metacognition and Learning. doi:10.1007/s11409-014-9129-8.

Bernacki, M. L., Nokes-Malach, T. J. N., \& Aleven, V. (2015). Examining self-efficacy during learning: Variability and relations to behavior, performance, and learning. Metacognition and Learning. doi:10. 1007/s11409-014-9127-x.

Binbarasan-Tüysüzoğlu, B., \& Greene, J. (2015). An investigation of the role of contingent metacognitive behavior in self-regulated learning. Metacognition and Learning. doi:10.1007/s11409-014-9126-y.

Boekaerts, M. (1997). Self-regulated learning: a new concept embraced by researchers, policy makers, educators, teachers, and students. Learning and Instruction, 7(2), 161-186.

Boekaerts, M. (1999). Self-regulated learning: where we are today. International Journal of Educational Research, 31(6), 445-457.

Boekaerts, M., \& Cascallar, E. (2006). How far have we moved toward the integration of theory and practice in self-regulation? Educational Psychology Review, 18(3), 199-210. doi:10.1007/s10648006-9013-.

Boekaerts, M., \& Corno, L. (2005). Self-regulation in the classroom: a perspective on assessment and intervention. Applied Psychology, 54(2), 199-231. 
Boekaerts, M., \& Niemivirta, M. (2000). Self-regulated learning: Finding a balance between learning goals and ego-protective goals. In M. Boekaerts, P. R. Pintrich, \& M. Zeidner (Eds.), Handbook of self-regulation (pp. 417-450). San Diego: Academic.

Bowers, E. P., von Eye, A., Lerner, J. V., Arbeit, M. R., Weiner, M. B., Chase, P., \& Agans, J. P. (2011). The role of ecological assets in positive and problematic developmental trajectories. Journal of Adolescence, 34(6), 1151-1165.

Bronfenbrenner, U. (1977). Toward an experimental ecology of human development. American Psychologist, $32(7), 513$.

Bronfenbrenner, U. (1994). Ecological models of human development. In M. Gauvain \& M. Cole (Eds.), Readings on the development of children (2nd ed., pp. 37-43). New York: Freeman.

Bronfenbrenner, U., \& Ceci, S. J. (1994). Nature-nurture reconceptualized in developmental perspective: A bioecological model. Psychological Review, 101(4), 568-586.

Butler, D. L., \& Winne, P. H. (1995). Feedback and self-regulated learning: a theoretical synthesis. Review of Educational Research, 65(3), 245-281.

Cleary, T. (2011). Emergence of self-regulated learning microanalysis: Historical overview, essential features, and implications for research and practice. In B. J. Zimmerman \& D. H. Schunk (Eds.), Handbook of self-regulation of learning and performance (pp. 329-345). New York: Routledge.

Corno, L. (1995). Comments on winne: analytic and systemic research are both needed. Educational Psychologist, 30(4), 201-206.

Dent, A. L. \& Hoyle, R. H. (2015). A framework for evaluating and enhancing alignment in self-regulated learning research. Metacognition and Learning. doi:10.1007/s11409-015-9136-4.

Dent, A. L., \& Koenka, A. C. (2015). The relation between self-regulated learning and academic achievement across childhood and adolescence: a meta-analysis. Educational Psychology Review (in press).

Efklides, A. (2011). Interactions of metacognition with motivation and affect in self-regulated learning: The MASRL model. Educational Psychologist, 46(1), 6-25. doi:10.1080/00461520.2011.53864.

Graesser, A., \& McNamara, D. (2010). Self-regulated learning in learning environments with pedagogical agents that interact in natural language. Educational Psychologist, 45(4), 234-244. doi:10.1080/00461520.2010. 51593.

Greene, J. A., \& Azevedo, R. (2007). Adolescents' use of self-regulatory processes and their relation to qualitative mental model shifts while using hypermedia. Journal of Educational Computing Research, $36(2), 125-148$.

Greene, J. A., \& Azevedo, R. (2009). A macro-level analysis of SRL processes and their relations to the acquisition of a sophisticated mental model of a complex system. Contemporary Educational Psychology, 34(1), 18-29. doi:10.1016/j.cedpsych.2008.05.00.

Greene, J. A., \& Azevedo, R. (2010). The measurement of learners' self-regulated cognitive and metacognitive processes while using computer-based learning environments. Educational Psychologist, 45(4), $203-209$. doi:10.1080/00461520.2010.51593.

Greene, J. A., Robertson, J., \& Costa, L. J. C. (2011). Assessing self-regulated learning using think-aloud methods. In B. J. Zimmerman \& D. H. Schunk (Eds.), Handbook of self-regulation of learning and performance (pp. 313-328). New York: Routledge.

Gress, C. L. Z., \& Hadwin, A. F. (2010). Advancing educational research on collaboration through the use of gStudy computer-supported collaborative learning (CSCL) tools: introduction to special issue. Computers in Human Behavior, 26, 785-786.

Gross, J. J. (2008). Emotion regulation. In M. Lewis, J. M. Haviland-Jones, \& L. F. Barrett (Eds.), Handbook of emotions (3rd ed., pp. 497-512). New York: Guilford Press.

Gross, J. J., \& John, O. P. (2003). Individual differences in two emotion regulation processes: implications for affect, relationships, and well-being. Journal of Personality and Social Psychology, 85, 348-362. doi:10. 1037/0022-3514.85.2.348.

Hoyle, R. H. (Ed.). (2010). Handbook of personality and self-regulation. Oxford: Wiley-Blackwell. doi:10.1002/ 9781444318111.

Järvelä, S., \& Hadwin, A. (2013). New frontiers: regulating learning in CSCL. Educational Psychologist, 48(1), 25-39. doi:10.1080/00461520.2012.74800.

Kaplan, A. (2004). Achievement goals and intergroup relations. Advances in Research on Motivation and Achievement, 13, 97-136.

Karabenick, S \& Zucho, A. (2015). Examining approaches to research on self-regulated learning: conceptual and methodological considerations. Metacognition and Learning. doi:10.1007/s11409-015-9137-3.

Karabenick, S. A., Woolleey, M. E., Friedel, J. M., Ammon, B. V., Blazevski, J., Bonney, C. R., De Groot, E., Gilbert, M. C., Musu, L., Kempler, T. M., \& Kelly, K. L. (2012). Cognitive processing of self-report items in educational research: do they think what we mean? Educational Psychologist, 42, 139-151. 
Kinnebrew, J. S., Biswas, G., Sulcer, B., \& Taylor, R. S. (2013a). Investigating self-regulated learning in teachable agent environments. In R. Azevedo \& V. Aleven (Eds.), International handbook of metacognition and learning technologies (pp. 451-470). New York: Springer New York. doi:10. 1007/978-1-4419-5546-3_2.

Kinnebrew, J. S., Loretz, K. M., \& Biswas, G. (2013b). A contextualized, differential sequence mining method to derive students' learning behavior patterns. Journal of Educational Data Mining, 5(1), 190-219.

Lichtinger, E., \& Kaplan, A. (2015). Employing a case study approach to capture motivation and self-regulation of young students with learning disabilities in authentic educational contexts. Metacognition and Learning. doi:10.1007/s11409-014-9131-1.

McCardle, L., \& Hadwin, A. F. (2015). Using multiple, contextualized data sources to measure learners' perceptions of their self-regulated learning. Metacognition and Learning. doi:10.1007/s11409-014-9132-0.

McCoy, D. C., Roy, A. L., \& Sirkman, G. M. (2013). Neighborhood crime and school climate as predictors of elementary school academic quality: a cross-lagged panel analysis. American Journal of Community Psychology, 52, 128-140.

Molenaar, I., \& Järvelä, S. (2014). Sequential and temporal characteristics of self and socially regulated learning. Metacognition and Learning, 9(2), 75-85.

Nathan, M. J., \& Alibali, M. W. (2010). Learning sciences. Wiley Interdisciplinary Reviews: Cognitive Science, 1(3), 329-345. doi:10.1002/wcs.54.

Newell, A. (1990). Unified theories of cognition. Cambridge: Harvard University Press.

Perry, N. E. (2002). Introduction: Using qualitative methods to enrich understandings of self-regulated learning. Educational Psychologist, 37(1), 1-3.

Perry, N. E., \& Rahim, A. (2011). Studying self-regulated learning in classrooms. In B. J. Zimmerman \& D. H. Schunk (Eds.), Handbook of self-regulation of learning and performance (pp. 122-136). New York: Routledge.

Pintrich, P. R. (2000). The role of goal orientation in self-regulated learning. In M. Boekarts, P. R. Pintrich, \& M. Zeidner (Eds.), Handbook of self-regulation: Theory, research and applications (pp. 451-502). San Diego: Academic.

Pintrich, P. R. (2004). A conceptual framework for assessing motivation and self-regulated learning in college students. Educational Psychology Review, 16(4), 385-407.

Pintrich, P. R., Smith, D. A., García, T., \& McKeachie, W. J. (1993). Reliability and predictive validity of the motivated strategies for learning questionnaire (MSLQ). Educational and Psychological Measurement, 53(3), 801-813.

Schmeichel, B. J., \& Baumeister, R. F. (2004). Self-regulatory strength. In R. F. Baumeister \& K. D. Vohs (Eds.), Handbook of self-regulation (pp. 84-98). New York: Guilford Press.

Schraw, G. (2010). Measuring self-regulation in computer-based learning environments. Educational Psychologist, 45, 258-266.

Sha, L., Looi, C. K., Chen, W., Seow, P., \& Wong, L. H. (2012). Recognizing and measuring self-regulated learning in a mobile learning environment. Computers in Human Behavior, 28(2), 718-728. doi:10.1016/j. chb.2011.11.01.

Winne, P. H. (1985). Steps toward promoting cognitive achievements. The Elementary School Journal, 85(5), 673-693. doi:10.1086/461429.

Winne, P. H. (1997). Experimenting to bootstrap self-regulated learning. Journal of Educational Psychology, 89(3), 397-410.

Winne, P. H. (2005). A Perspective on state-of-the-art research on self-regulated learning. Instructional Science, 33(5), 559-565.

Winne, P. H. (2010). Improving measurements of self-regulated learning. Educational Psychologist, 45(4), 267276. doi: $10.1080 / 00461520.2010 .51715$.

Winne, P. H. (2011). A cognitive and metacognitive analysis of self-regulated learning. In B. J. Zimmerman \& D. H. Schunk (Eds.), Handbook of self-regulation of learning and performance (pp. 15-32). New York: Routledge.

Winne, P. H. (2014). Issues in researching self-regulated learning as patterns of events. Metacognition and Learning, 9(2), 229-237.

Winne, P. H., \& Baker, R. S. (2013). The potentials of educational data mining for researching metacognition, motivation and self-regulated learning. Journal of Educational Data Mining, 5(1).

Winne, P. H., \& Hadwin, A. F. (1998). Studying as self-regulated learning. In D. J. Hacker, J. Dunlosky, \& A. C. Graesser (Eds.), Metacognition in educational theory and practice (pp. 277-304). Hillsdale: Erlbaum.

Winne, P. H., \& Hadwin, A. F. (2008). The weave of motivation and self-regulated learning. In D. Schunk \& B. J. Zimmerman (Eds.), Motivation and self-regulated learning: Theory, research, and applications (pp. 297314). Mahwah: Erlbaum. 
Winne, P. H., \& Hadwin, A. F. (2013). NStudy: Tracing and supporting self-regulated learning in the internet. In R. Azevedo \& V. Aleven (Eds.), International handbook of metacognition and learning technologies (pp. 293-308). New York: Springer. doi:10.1007/978-1-4419-5546-3 2.

Winne, P. H., \& Perry, N. E. (2000). Measuring self-regulated learning. In M. Boekaerts, P. R. Pintrich, \& M. Zeidner (Eds.), Handbook of self-regulation (pp. 531-566). San Diego: Academic.

Winne, P. H., Zhou, M., \& Egan, R. (2011). Designing assessments of self-regulated learning. In G. Schraw \& D. H. Robinson (Eds.), Assessment of higher order thinking skills (pp. 89-118). Charlotte: Information Age.

Wirth, J., \& Leutner, D. (2008). Self-regulated learning as a competence: implications of theoretical models for assessment methods. Zeitschrift Für Psychologie/Journal of Psychology, 216(2), 102. doi:10.1027/00443409.216.2.102.

Wolters, C. A., \& Pintrich, P. R. (1998). Contextual differences in student motivation and self-regulated learning in mathematics, English, and social studies classrooms. Instructional Science, 26(1-2), 27-47.

Zimmerman, B. J. (1989). A social cognitive view of self-regulated academic learning. Journal of Educational Psychology, 81(3), 329.

Zimmerman, B. J. (2000). Attaining self-regulation: A social cognitive perspective. In M. Boekaerts, P. R. Pintrich, \& M. Zeidner (Eds.), Handbook of self-regulation (pp. 13-39). San Diego: Academic.

Zimmerman, B. J., \& Schunk, D. H. (2008). Motivation: An essential dimension of self-regulated learning. In B. J. Zimmerman \& D. H. Schunk (Eds.), Motivation and self-regulated learning: Theory, research, and applications (pp. 1-30). New York: Routledge.

Zimmerman, B. J., \& Schunk, D. H. (2011). Handbook of self-regulation of learning and performance. New York: Taylor \& Francis. 